\title{
Specific Injuries Management in the Postoperative of Congenital Heart Diseases (II): Univentricular Hearts
}

\author{
A. Sánchez Andrés ${ }^{1}$, C. González Miño르, E. Valdés Diéguez ${ }^{3}$, L. Boni ${ }^{3}$, J. I. Carrasco Moreno' \\ ${ }^{1}$ Pediatric Cardiology Unit, Hospital Universitario y Politécnico La Fe, Valencia, Spain \\ ${ }^{2}$ Pediatric Intensive Care Unit, Hospital General de Castellón, Castellon, Spain \\ ${ }^{3}$ Pediatric Surgery Unit, Hospital Universitario y Politécnico La Fe, Valencia, Spain \\ Email: tonisanchan@hotmail.com
}

Received 27 February 2015; accepted 12 March 2015; published 17 March 2015

Copyright (C) 2015 by authors and Scientific Research Publishing Inc.

This work is licensed under the Creative Commons Attribution International License (CC BY).

http://creativecommons.org/licenses/by/4.0/

c) (i) Open Access

\begin{abstract}
It is very important to understand that the univentricular heart surgery is just palliative, not being in anyway a definitive or curative surgery, but nowadays it's the best initial treatment of this complex heart disease. The fundamental philosophy of treatment of every univentricular heart is to ensure the flow system and/or restrict the lung flow. Thus, initially a patient with univentricular heart who is undergoing surgery may need to ensure systemic flow (reconstruction of the aortic arch type Norwood), to restrict the lung flow (pulmonary banding) or to provide enough pulmonary flow (pulmonary-systemic fistulae). However, some heart diseases with univentricular physiology remain "balanced" autonomously, until the "second" stage of palliation is performed (cavo-pulmonary anastomosis type Glenn), but others require performance of pulmonary banding, if there's no native lung protection and/or repair of the systemic circuit in a first stage, to reach next palliation steps in the best possible conditions.
\end{abstract}

\section{Keywords}

Univentricular heart, Postoperative, Congenital heart disease, Norwood, Glenn, Fontan

\section{Introduction}

Biventricular circulatory system consists of a double circuit (pulmonar and systemic) connected in series through blood flows driven by two distinct, well developed ventricular chambers which have enough contractile power to act as pumps. From an anatomical segmental view, the heart must then be essentially a biventricular atrioventricular connection with two atrioventricular orifices: normal, permeable and competent.

How to cite this paper: Sánchez Andrés, A., González Miño, C., Valdés Diéguez, E., Boni, L. and Carrasco Moreno, J.I. (2015) Specific Injuries Management in the Postoperative of Congenital Heart Diseases (II): Univentricular Hearts. Open Journal of Pediatrics, 5, 67-75. http://dx.doi.org/10.4236/ojped.2015.51012 
Certain complex cardiac malformations are characterized by only a functionally useful ventricular chamber to keep the requirement to simultaneously hold the circulation in both circuits (pulmonary and systemic) when they are connected in parallel as occurs after birth. For identification, colloquially the name "univentricular heart" or "single ventricle" is used, even if it's actually possible to verify in many cases the presence of two ventricles regardless of the absolute size of each. The vast majority of these hearts have a univentricular atrioventricular connection, and the classic example is the tricuspid atresia. It is noted, that there are certain malformations with two well developed ventricles in which a biventricular surgical correction is impossible, so in these cases it is necessary to resort to univentricularization.

The postnatal circulation of cardiac malformations with univentricular context involves two fundamental problems: volume overload of the single ventricle and systemic desaturation; this is because both circuits, pulmonary and systemic flow are receiving the blood flow simultaneously in parallel. From the perspective of hemodynamics, the result of the surgery is a univentricular circulatory model number, even if the transaction has a marked palliative.

The application of mitigation techniques in such patients, atrioseptostomy Rashkind, surgical atrioseptectomy (Blalock-Hanlon), and those aimed to regulate the pulmonary blood flow (aortopulmonary anastomosis and pulmonary artery banding), projected a ray of hope on these malformations (there are cases of prolonged survival with these techniques). Throughout these years there have been other variants of surgical intervention called "repair" that, when applied appropriately respond to this group of patients with previous or palliative surgery have helped reduce mortality significantly, these are veno-atrium-pulmonary shunt techniques.

The main objective of this article is to provide a list of the most common surgical procedures performed trough the stages of palliation for univentrcular hearts, along with the possible complications to be expected in the postoperative period. Some recommendations as to their diagnoses and management are also discussed.

\section{Perioperative Management of Univentricular Hearts}

Univentricular heart diseases have different physiology depending on stage of palliation in which they are (Table 1 and Table 2). Following the procedures that are performed, either systemic-pulmonary shunt or pulmonary artery banding, the relationship between pulmonary and systemic blood flow (Qp:Qs) depends on the systemicvascular resistance, the size of the pulmonary artery shunt and to a lesser degree, of pulmonary vascular resistance. The

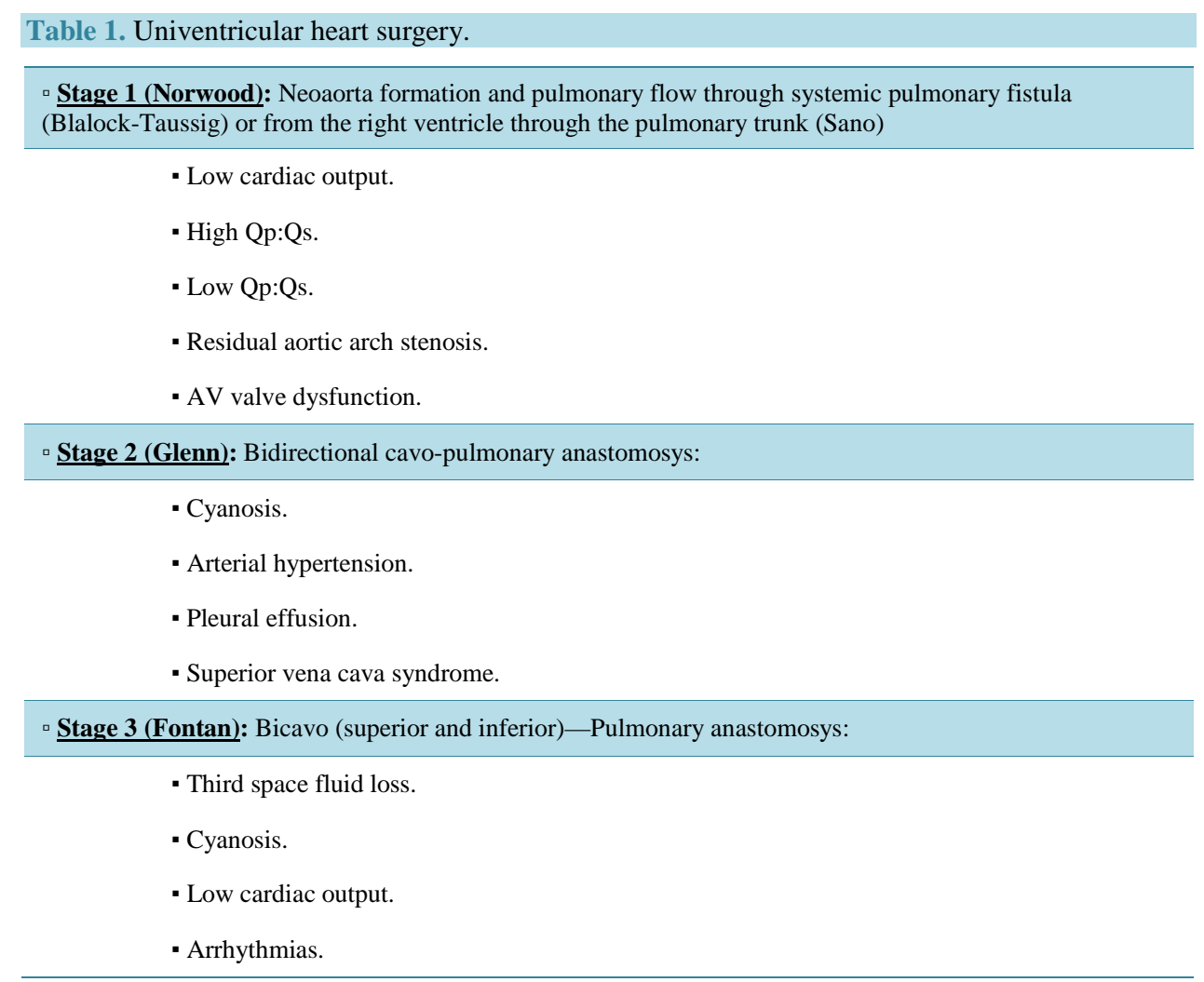


Table 2. Palliative procedures.

\begin{tabular}{l}
\hline Systemic to Pulmonary Shunts (Blalock-Taussig, Modified Blalock-Taussig, Central Fistulae) \\
\hline - Excessive pulmonary flow. \\
- Arterial Hypotension. \\
- Thrombosis. \\
\hline Pulmonary Artery Banding \\
\hline - Cyanosis. \\
- Excessive pulmonary flow.
\end{tabular}

arterial saturation is definitely half between systemic and pulmonary venous saturations, weighted by the Qp:Qs, therefore, any circumstance that produces a decrease in venous admixture, pulmonary venous saturation or Qp:Qs, can produce increases in the degree of cyanosis. The problems in the immediate postoperative period usually lead mainly to a decrease in oxygen delivery to tissues due to low cardiac output and/or excessive level of cyanosis, so it is of vital importance to determine if the problem is related to low heart output, an unequal distribution of the cardiac output (increases or decreases in Qp:Qs) or problems in pulmonary venous saturation. Poor systemic perfusion or high difference in arterio-venous mixing suggests a problem related to low output or increased Qp:Qs. In contrast, the hemodynamic maintenance cyanosis suggests a primary lung problem or decrease in Qp:Qs.

One of the most important principles to consider during the postoperative management of heart disease with univentricular physiology is that increases in systemic vascular resistance can increase blood pressure, Qp:Qs and arterial saturation at the expense of systemic perfusion. Therefore, adequate blood pressure should not be taken itself as a sign of adequate systemic perfusion. Many of the current studies suggest that high blood pressure is actually associated with low tissue oxygen delivery. Hence, the use of afterload reducing agents such as milrinone, nitroprusside or phenoxybenzamine in order to improve systemic perfusion is becoming widespread. Blood pressure can be maintained within acceptable ranges by improving cardiac output with the use of low doses of epinephrine or norepinephrine.

Another goal in the management of these patients is to maximize cardiac output to ensure adequate tissue oxygenation and to assess those adjustments that can be made by manipulating the ratio Qp:Qs. Again, this principle supports the use of afterload reducing agents that can increase cardiac output and counteract pulmonary circulatory overload. Therapeutic strategies aimed at increasing pulmonary vascular resistance have their limitations in practice, overall because the mayor component of pulmonary resistance takes place where shunt or banding have been made.

The first stage of palliative surgery or Norwood procedure is one of the leading major efforts to palliate the hypoplastic left heart syndrome (HLHS), but operative mortality remains worrisome. Several factors associated with poor prognosis have been described, such as preoperative hemodynamic instability, the diminutive size of the ascending aorta, the commitment of the ventricular function, significant tricuspid regurgitation, prematurity and low birth weight. Instead, it is a good prognostic factor type of diagnosis, being more favorable, for example, those diseases that do not correspond to HLHS. For this reason, some groups considered a contraindication to perform this operation, at least at this early stage, prematurity, weight below $2.5 \mathrm{~kg}$, significant ventricular dysfunction, significant tricuspid regurgitation, the presence of anomalous pulmonary venous drainage total obstruction and the presence of other severe associated malformations. These conditions can contraindicate surgery up to $25 \%$ of patients with this diagnosis [1] [2].

Currently, most diagnoses are made in the antenatal period, what constitutes a significant contribution in the management strategy of these children. HLHS antenatal diagnosis can be made from the eighteenth week of pregnancy, allowing to properly plan patient's birth and timely implement measures to stabilize the patient hemodynamics, including early onset of prostaglandins or early and scheduled completion of the Rashkind maneuver if there were any restriction at the level of the fossa ovalis. Note that this disease can go unnoticed initially and the patient may present with cardiogenic shock after a few days of life, the result of spontaneous closure of the ductus.

Many groups continue to perform what is known as "classic Norwood", because the reconstruction of the as- 
cending aorta and arch with homograft offers better results in terms of lower incidence of recoarctation and less distortion of the pulmonary valve in comparison with other techniques. However, these differences are not so clear so it is permissible to choose the technique with which the surgical team feels most comfortable. Other groups have designed variants tailored for each individual case, but that conceptually belong to the same intervention and therefore have been arbitrarily called "modified Norwood". Patients who develop postoperative gradient at the aortic arch usually correspond to "classical” repairs, because of redundancy of the patch, which can generate an invagination thereof. The use of "cardiac arrest” is an interesting technical contribution in recent years, aimed at reducing the cerebral ischemia time. Its routine implementation in the Norwood operation can help to preserve brain function in the medium and long term.

The reported perioperative mortality figures have historically been around $20 \%$ - 30\%. The best series have managed to lose $10 \%$ but others reach more than 50\%. Bartram et al performed a detailed analysis of the post Norwood causes of death, being coronary irrigation problems and those derived from the most common shunt the main causes. The initial mortality of this series is comparable to international studies, however, it has the important limitation of being a small series. It is not a minor event that these patients remain with abnormal physiology, with pulmonary flow dependent on a systemic-pulmonary shunt, so the intra and postoperative management is a real challenge.

Several methods have been gradually incorporated to optimize postoperative management, such as adding carbon dioxide or nitrogen to air mixture, continuous measurement of mixed venous saturation and, recently, the use of phenoxybenzamin (POB) with promising results. The principle of the use of phenoxybenzamin is the increase in systemic cardiac output by maximal dilatation of the systemic circulation. This effect results in a more stable parallel circulation through prevention of fluctuations in systemic vascular resistance in the early postoperative period. However, there is a technical amendment introduced lately, which seems to contribute significantly in reducing mortality and facilitate the handling in the intensive care unit, because the balance between pulmonary and systemic circulation ceases to be critical. This modification is to replace the Blalock-Taussig shunt (Figure 1) with one that connects the ventricle and the pulmonary artery (Figure 2), so as to obtain only systolic flow, rather than systolic and dyastolic as in the first situation. While there are some problems in relation to this option, popularized by Sano et al, it seems to be one of the most important contributions of recent times and it seems reasonable to consider its use selectively. The risk of death continues in the medium term, especially before the Glenn operation. There is unpublished evidence that the Sano modification could also help to reduce this attrition before the second stage [3] [4].

The second and the third stage in palliation of univentricular hearts are unique from the standpoint of pulmonary blood flow becoming dependent on non-pulsatile venous flow. The bidirectional cavopulmonary shunt, the classic Glenn anastomosis (Figure 3), by virtue of increasing the effective pulmonary flow improves the systemic arterial oxygen saturation, volume unloads the ventricle, and also alters the ventricular geometry, whether the ventricle is of right or left ventricular morphology [5]. One of the mechanisms by which the bidirectional cavopulmonary connection improves the systemic arterial oxygen saturation is to increase the so-called effective pulmonary blood flow [6].

A particular aspect in bidirectional cavo-pulmonary anastomosis is that the pulmonary flow is dependent heavily on the strength of two distinct vascular beds. Both circulations, the brain and lung, have opposite responses to changes in the levels of carbon dioxide $\left(\mathrm{CO}_{2}\right)$, acid-base balance and oxygen. This can cause particular difficulties in the treatment of elevated pulmonary vascular resistance or low arterial oxygen saturation. Hyperventilation and alkalosis, for example, have limited utility in this aspect, as though they are effective pulmonary vasodilators, the hyperventilation and alkalosis produce cerebral vasoconstriction. Because pulmonary blood flow is dependent on venous return through the superior vena cava (largely composed of cerebral blood flow), maneuvers that cause decreased cerebral blood flow, may decrease the pulmonary blood flow and increase the degree of hypoxemia. Hyperventilation after the completion of a Glenn produces in fact a decrease in cerebral blood flow and thus a decrease in arterial oxygen saturation [7]. Other techniques commonly used to decrease pulmonary vascular resistance as the deep sedative drug can also cause a decrease in cerebral blood flow. Inhaled NO [8], which acts selectively on the pulmonary vessels has proven to be an effective measure in reducing the transpulmonary pressure gradient in patients undergoing such surgery Glenn and thus work in combination with moderate hypoventilation in case of high pulmonary vascular resistance and cyanosis. When the degree of cyanosis is not excessive, expectant attitude with adequate hemodynamic support and maintenance of hemoglobin levels within normal limits, may be sufficient. This is because the saturation tends to improve gradually in the first days after surgery and again at the 


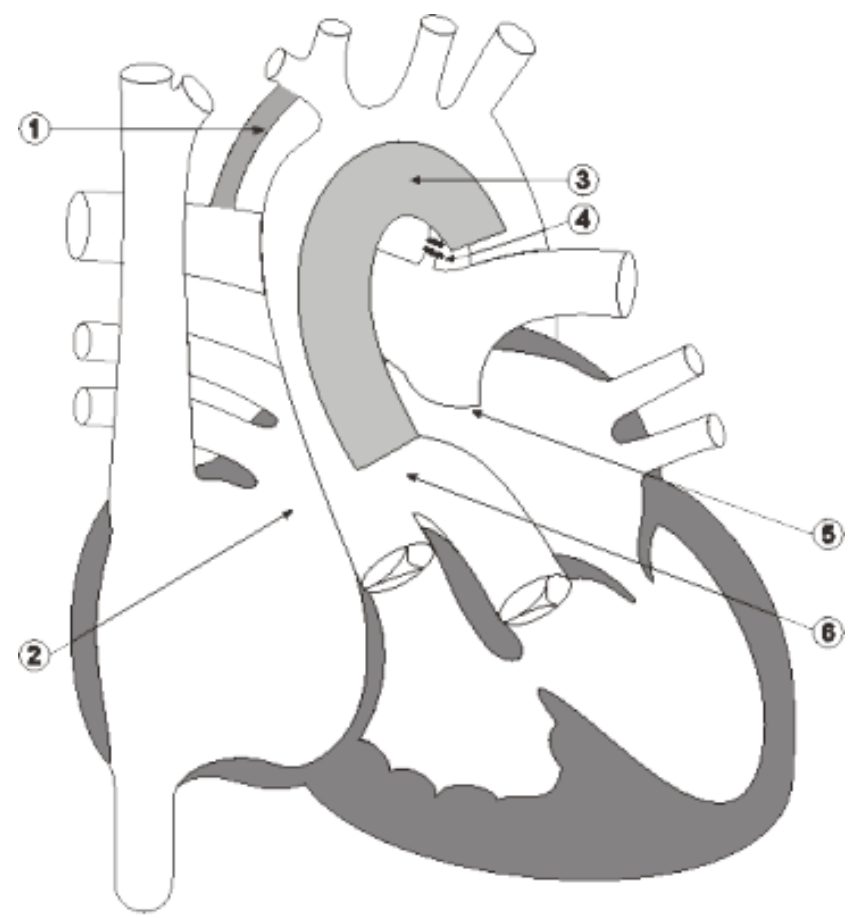

Figure 1. Norwood repair of the aortic arch with systemicpulmonary shunt (modified Blalock-Taussig). 1. BT shunt. 2. Atrioseptectomy. 3. Norwood arch repair. 4. Clips on ductus. 5. Pulmonary trunk section. 6. Neo-aorta (Native pulmonary trunk with small native aorta).

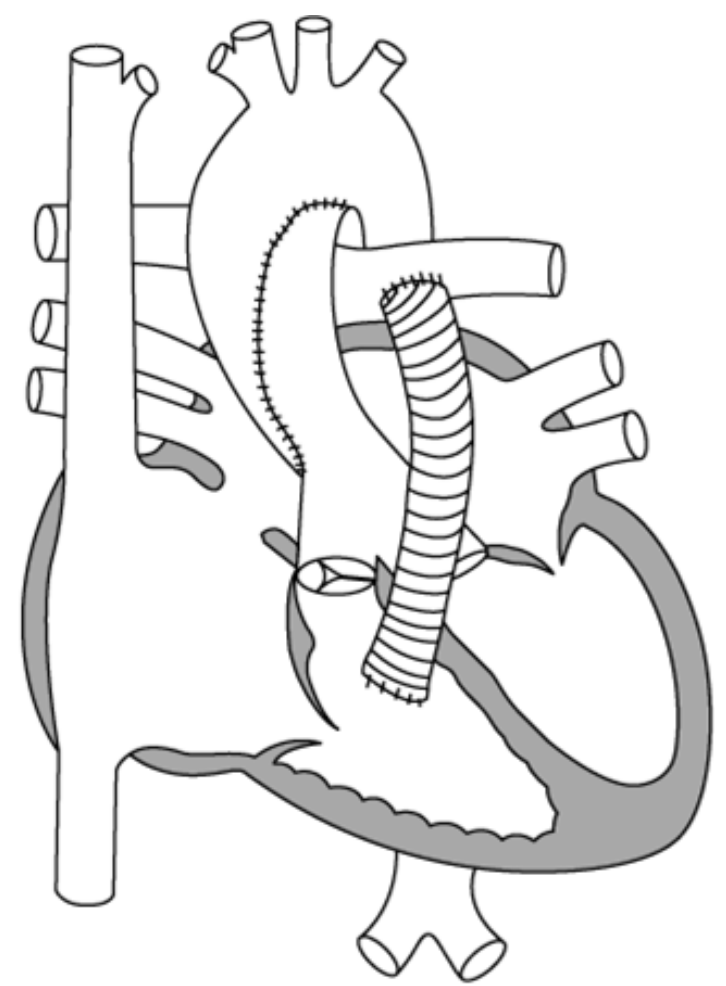

Figure 2. Norwood repair of the Aortic arch with right ventricle to pulmonary bifurcation conduit (Sano’s conduit). 


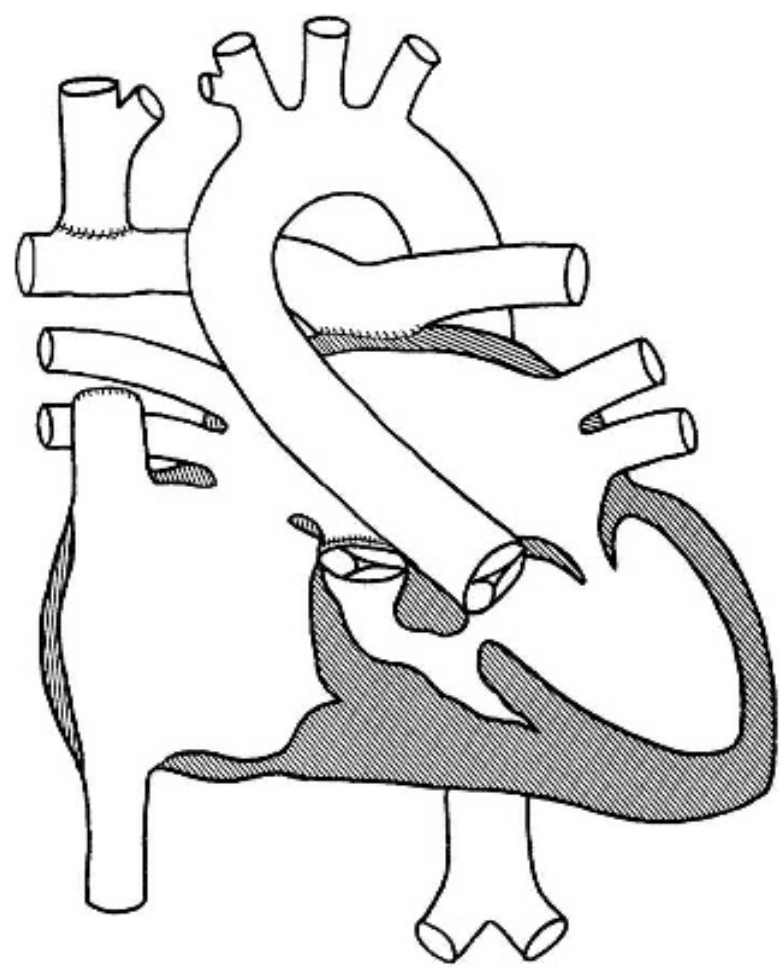

Figure 3. Superior cavo-pulmonary connection (Bidireccional Glenn).

time of extubation, provided that there are no problems in the lung or airways. Persistent cyanosis, can produce a series of problems such as decompression of veno-venous collaterals that divert the flow of the superior vena cava away from the pulmonary circulation.

Systemic hypertension is a common phenomenon after such interventions Glenn. This could occur in response to increased cerebral venous pressure or an improvement in the cost of a ventricle that is volume unloaded. Treatment with vasodilators is often necessary and blood pressure tends to decrease to normal range in the first days after surgery, a proportion of patients requiring treatment with ACE inhibitors for long periods of time [9].

The presence of residual shunts and aorto-pulmonary collaterals can be problematic in the post-Glenn. They have been associated with the presence of persistent pleural effusions, elevated venous pressures and low cardiac output. It is very important to recognize that ventricular structure suffers so many changes with this type of intervention, mainly because it reduces left to right shunts, particularly with the bidirectional Glenn. When systemic flow is dependent on the flow through a ventricular septal defect or bulbo-ventricular foramen, sharp decreases in ventricular dimensions may precipitate the onset of subaortic stenosis. The emergence of an ejection murmur in a patient with susceptible anatomy after the completion of a bidirectional Glenn should lead to screening for this injury [10].

The physiology of the Fontan circulation is a mixture between the bidirectional Glenn and normal cardiovascular physiology. As in Glenn, pulmonary blood flow is dependent on the systemic venous pressure, and all pulmonary flow is effective. However, pulmonary and systemic circulations are well differentiated, as in the normal heart. If circulation is composed of a Fontan fenestration in the atrial septum [11] [12], there may be varying degrees of left-right shunt causing a mild systemic desaturation. In this unique circulation, the two principal determinants of long-term outcome are low pulmonary vascular resistance (PVR) and adequate singleventricle myocardial function. Drugs that lower PVR and/or improve myocardial performance could optimize circulatory efficiency and potentially improve outcomes [13] [14].

Major issues for intensivists arise when there is elevation of pulmonary arterial pressure, as this can occur either because the pulmonary vascular resistance is elevated, mechanical obstruction in the pulmonary artery or when there is myocardial dysfunction causing an increase in the pressure on pulmonary veins to left atrium level [14]. Numerous studies have shown that high pressures in the pulmonary artery ( $>10-15 \mathrm{mmHg})$ are associated with poor prognosis in patients undergoing Fontan surgery, mainly because it is very difficult to maintain central 
venous pressures in these values without producing output of the third space fluid. When this loss of fluids progresses, patients develop pleural effusions, ascites and peripheral edema. It becomes necessary then to increase the pressure of the respirator to maintain adequate functional residual capacity and an adequate tidal volume against a distended abdomen, a heavy chest and small effective pleural lung distension. The increases in airway pressures, particularly in the absence of pulmonary parenchymal disease, increase pulmonary vascular resistance, and therefore even higher venous pressures are needed to maintain cardiac output. Also, when intraabdominal pressure increases, renal perfusion pressure decreases, specially when there is low cardiac output or blood pressure is at the lower limit, what is common in these circumstances. In general, the fenestration in the Fontan surgery can reduce the risk of some of these complications by providing a source of systemic flow which is not dependent on passage through the pulmonary circulation, but can also cause a decrease in pulmonary arterial pressure sufficient to reduce the loss of third space fluid [15].

When a patient with Fontan circulation physiology is in a state of low cardiac output, it is essential to identify and treat the underlying cause. It is very common for patients during the Fontan postoperative period, to require large amounts of volume on the first day after surgery.

The persistence of low central pressures strongly suggests the need of volume. The obstruction of the pulmonary artery should be considered as the cause of low cardiac output when left atrial pressure is low and central venous pressure is high. If the central venous pressure was not monitored, the heavy losses of third space fluid with low or normal pressures in the left atrium should increase the degree of suspicion. Even in the presence of fenestration, its ability to maintain cardiac output against an anatomical or physiological obstruction to pulmonary blood flow is significantly limited compared to the situation that occurs after the Glenn. Therefore, a limited pulmonary blood flow leads to a situation of low cardiac output and when there is fenestration significant cyanosis. Cyanosis may also be observed when there are intrapulmonary arteriovenous malformations (such as after the Glenn) or due to ventilation-perfusion imbalance that occurs in these situations of low cardiac output [16].

If the high pulmonary vascular resistance is responsible for the elevation of central venous pressure it is indicated to start with conventional treatments as supplemental oxygen, hyperventilation and alkalosis. As in patients with bidirectional Glenn, the use of high positive pressure during ventilation to achieve these ends may be counterproductive. To avoid high pressure ventilation may increase the minute volume and cardiac output and high frequency ventilation may decrease the $\mathrm{PaCO} 2$ at low airway pressures [13]. Intravenous vasodilators such as prostacyclin or prostaglandin E, should be used with caution, because of the risk of systemic vasodilatation with limited cardiac output. Inhaled NO has been referred to in several studies as an effective drug to lower the transpulmonary pressure gradient [8].

The presence of a low cardiac output with high central venous pressure in left atria, indicates dysfunction in patients with Fontan circulation. Myocardial dysfunction may occur during ischemia-reperfusion period, when aortic clamping and cardioplegia used to create the connection. You may also be related to poor preoperative myocardial function. The only effective therapy for low cardiac output due to ventricular dysfunction Fontan intervention is to improve cardiac output by inotropic agents and reduce the pressure in the left atrium. The use of inotropic agents such as phosphodiesterase inhibitors, dobutamine or low doses of epinephrine (less than 0.05 microgrs $/ \mathrm{kg} /$ minute) may be helpful. If systemic blood pressure could tolerate it, an aggressive reduction of afterload with vasodilating agents (ONi in short term and sildenafil in long term [14]) may also reduce left atrial pressure in a meaningful way. If there is good reason to believe that the ventricular dysfunction is reversible, mechanical circulatory support (ECMO) could also be an effective therapy [15]. Because in those with persistent Fontan aorto-pulmonary collaterals may be associated with hemodynamic status similar to those with left ventricular dysfunction, aggressive evaluation and embolization of these collateral could be useful in these situations [17].

\section{Conclusions}

"Univentricular heart" denotes a wide variety of rare and complex congenital cardiac malformations whereby both atria predominantly egress into a functional single ventricle. Although most patients will be managed by a staged surgical approach in view of an ultimate Fontan procedure, a minority will not undergo Fontan palliation either because they maintain reasonably balanced systemic and pulmonary circulations or as a result of unfavorable hemodynamics.

Following major improvements of the surgical technique, modified Fontan operations represent a well accepted treatment for separation of pulmonary and systemic circulation in children with functionally univentricular hearts. Optimisation of flow dynamics was achieved by introduction of total cavopulmonary 
anastomosis, which can be performed either as a lateral tunnel (Figure 4) or as an extracardiac conduit procedure (Figure 5). Staging the Fontan procedure by a bidirectional cavopulmonary anastomosis with early relief

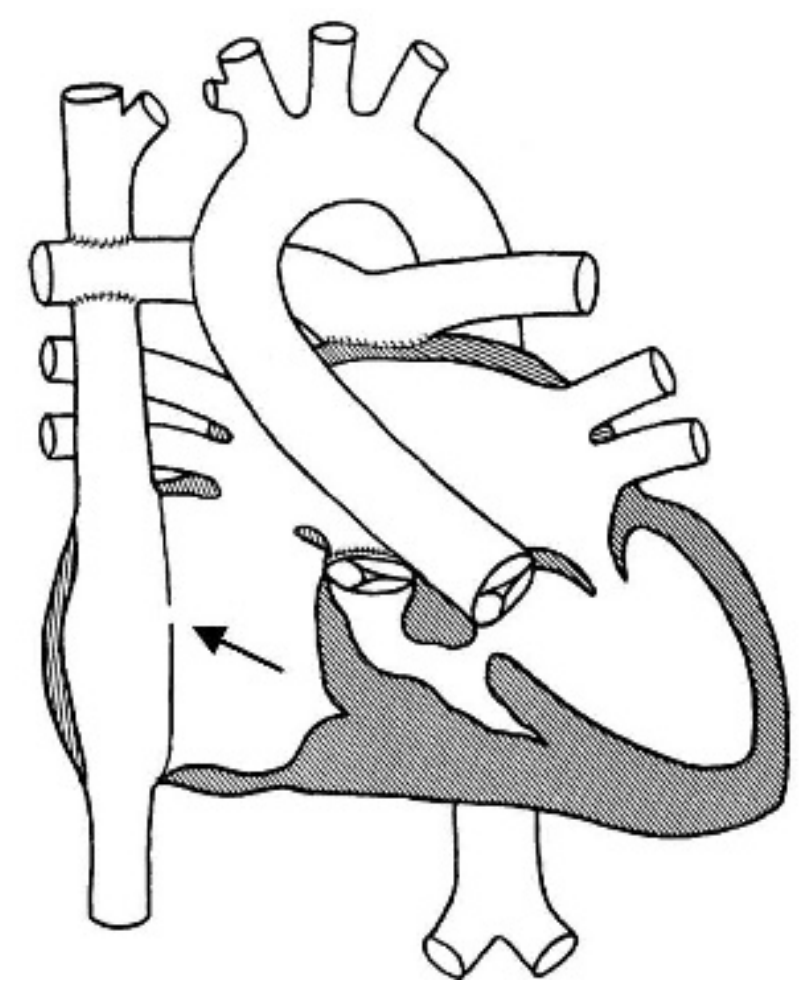

Figure 4. Superior and inferior cavo-pulmonary connection with lateral tunnel (Intracardiac Fontan).

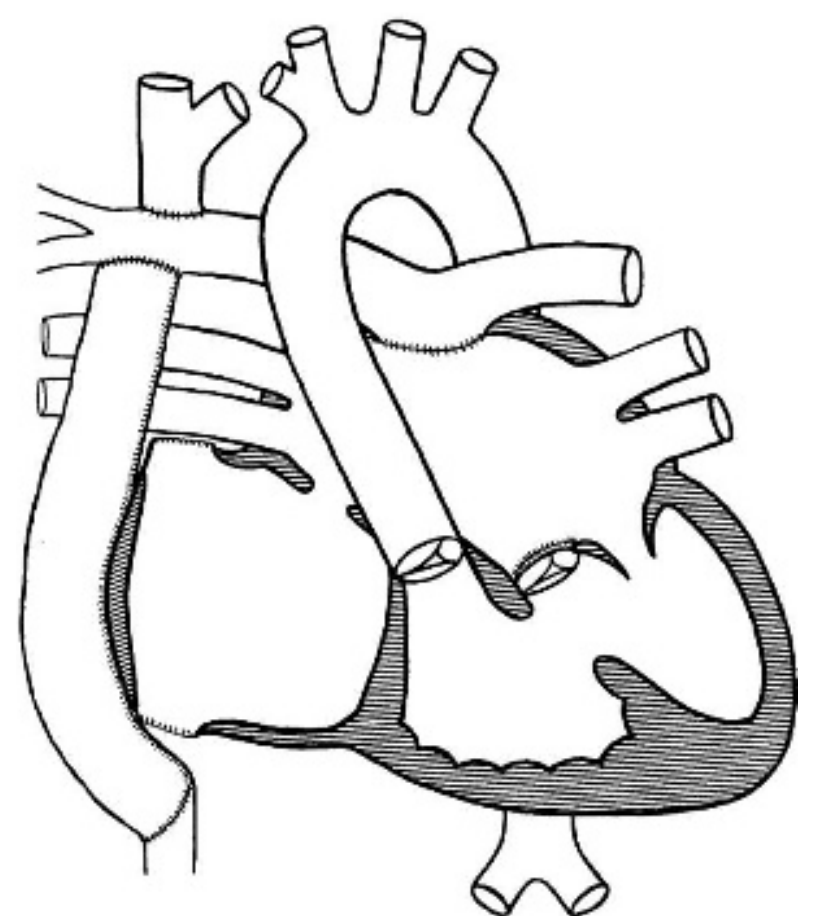

Figure 5. Superior and inferior cavo-pulmonary connection with extracardiac conduit (Extracardiac Fontan). 
of volume load on the single ventricle and later completion of the Fontan circulation reduced the perioperative risk. Despite reduced operative mortality there remains a worrying decrement during long term follow up due to sequelae and complications of the univentricular circulation [18].

\section{References}

[1] Khairi, P., Poirier, N. and Mercier, L.-A. (2007) Congenital Heart Desease for the Adult Cardiologist. Univentricular Heart. Circulation, 115, 800-812.

[2] De Oliveira, N.C., Ashburn, D.A., Khalid, F., et al. (2004) Prevention of Early Sudden Circulatory Collapse after the Norwood Operation. Circulation, 110, II133-II138. http://dx.doi.org/10.1161/01.CIR.0000138399.30587.8e

[3] Hoffman, G.M., Tweddell, J.S., Ghanayem, N.S., et al. (2004) Alteration of the Critical Arteriovenous Oxygen Saturation Relationship by Sustained Afterload Reduction after the Norwood Procedure. Journal of Thoracic and Cardiovascular Surgery, 127, 738-745. http://dx.doi.org/10.1016/S0022-5223(03)01315-1

[4] Chang, A.C., Hanley, F.L., Wernovsky, G., et al. (1993) Early Bidireccional Cavopulmonary Shunt in Young Infants. Postoperative Course and Early Results. Circulation, 88, II149-II158.

[5] Berdat, P.A., Belli, E., Lacour-Gayet, F., Planché, C. and Serraf, A. (2005) Additional Pulmonary Blood Flow Has No Adverse Effect on Outcome after Bidirectional Cavopulmonary Anastomosis. The Annals of Thoracic Surgery, 79, 2937. http://dx.doi.org/10.1016/j.athoracsur.2004.06.002

[6] Freedom, R.M., Nykanen, D. and Benson Lee, N. (1998) The Physiology of the Bidirectional Cavopulmonary Connection. The Annals of Thoracic Surgery, 66, 664-667. http://dx.doi.org/10.1016/S0003-4975(98)00618-3

[7] Bradley, S.M., Simsic, J.M. and Mulvihill, D.M. (1998) Hyperventilation Impairs Oxygenation after Bidirectional Superior Cavopulmonary Connection. Circulation, 98, II372-II376.

[8] Gamillscheg, A., Zobel, G., Urlesberger, B., et al. (1997) Inhaled Nitric Oxide in Patients with Critical Pulmonary Perfusion after Fontan-Type Procedures and Bydireccional Glenn Anastomosis. Journal of Thoracic and Cardiovascular Surgery, 113, 435-442. http://dx.doi.org/10.1016/S0022-5223(97)70355-6

[9] Lee, T.M., Aiyagari, R., Hirsch, J.C., Ohye, R.G., Bove, E.L. and Devaney, E.J. (2012) Risk Factor Analysis for Second-Stage Palliation of Single Ventricle Anatomy. The Annals of Thoracic Surgery, 93, 614-619. http://dx.doi.org/10.1016/j.athoracsur.2011.10.012

[10] Alsoufi, B., et al. (2012) Current Outcomes of the Glenn Bidirectional Cavopulmonary Connection for Single Ventricle Palliation. European Journal Cardio-Thoracic Surgery, 72, 42-49. http://dx.doi.org/10.1093/ejcts/ezr280

[11] Bridges, N.D., Lock, J.E. and Castaneda, A.R. (1990) Baffle Fenestration with Subsequent Transcatheter Closure. Modification of the Fontan Operatión for Patients at Increased Risk. Circulation, 82, 1681-1689. http://dx.doi.org/10.1161/01.CIR.82.5.1681

[12] Lemler, M.F., Scott, W.A., Leonard, S.R., et al. (2002) Fenestration Improves Clinical Outcome of the Fontan Procedure. Circulation, 105, 207-212. http://dx.doi.org/10.1161/hc0202.102237

[13] Shekerdemian, L.S., Bush, A., Shore, D.F., et al. (1997) Cardiopulmonary Interaction after Fontan Operations: Augmentation of Cardiac Output Using Negative Pressure Ventilation. Circulation, 96, 3934-3942. http://dx.doi.org/10.1161/01.CIR.96.11.3934

[14] Tunks, R.D., et al. (2014) Sildenafil Exposure and Hemodynamic Effect after Fontan Surgery. Pediatric Critical Care Medicine, 15, 28-34. http://dx.doi.org/10.1097/PCC.0000000000000007

[15] Kaulitz, R. and Hofbeck, M. (2005) Current Treatment and Prognosis in Children with Functionally Univentricular Hearts. Archives of Disease in Childhood, 90, 757-762. http://dx.doi.org/10.1136/adc.2003.034090

[16] Spicer, R.L., Uzark, K.C., Moore, J.W., et al. (1996) Aortopulmonary Collateral Vessels and Prolonged Pleural Effusions after Modified Fontan Procedures. American Heart Journal, 131, 1164-1168. http://dx.doi.org/10.1016/S0002-8703(96)90092-7

[17] Triedman, J.K., Bridges, N.D., Mayer, J.E.J., et al. (1993) Prevalence and Risk Factors for Aortopulmonary Collateral Vessels after Fontan and Bidireccional Glenn Procedures. Journal of the American College of Cardiology, 22, 207215.

[18] Lastinger, L. and Zaidi, A.N. (2013) The Adult With a Fontan: A Panacea without a Cure? Circulation Journal, 77, 2672-2681. http://dx.doi.org/10.1253/circj.CJ-13-1105 